\title{
Nonlinear Conditions for Ultradifferentiability
}

\author{
David Nicolas Nenning ${ }^{1} \cdot$ Armin $^{\text {Rainer }^{1}}{ }^{\text {D }} \cdot$ Gerhard Schindl $^{1}$
}

Received: 24 February 2021 / Accepted: 4 June 2021 / Published online: 19 June 2021

(c) The Author(s) 2021

\begin{abstract}
A remarkable theorem of Joris states that a function $f$ is $C^{\infty}$ if two relatively prime powers of $f$ are $C^{\infty}$. Recently, Thilliez showed that an analogous theorem holds in Denjoy-Carleman classes of Roumieu type. We prove that a division property, equivalent to Joris's result, is valid in a wide variety of ultradifferentiable classes. Generally speaking, it holds in all dimensions for non-quasianalytic classes. In the quasianalytic case we have general validity in dimension one, but we also get validity in all dimensions for certain quasianalytic classes.
\end{abstract}

Keywords Joris theorem - Division property · Ultradifferentiable classes ·

(Non-)Quasianalytic · Holomorphic approximation · Almost analytic extension

Mathematics Subject Classification 26E10 $\cdot 30 \mathrm{E} 10 \cdot 32 \mathrm{~W} 05 \cdot 46 \mathrm{E} 10 \cdot 46 \mathrm{E} 25 \cdot 58 \mathrm{C} 25$

\section{Introduction}

A remarkable Theorem of Joris [11, Théorèm 2] states: if $f: \mathbb{R} \rightarrow \mathbb{R}$ is a function and $p, q$ are relatively prime positive integers, then

$$
f^{p}, f^{q} \in C^{\infty} \Longrightarrow f \in C^{\infty} \text {. }
$$

Since smoothness can be tested along smooth curves by a theorem of Boman [3], one immediately infers that the implication (1) holds on arbitrary open subsets of $\mathbb{R}^{d}$,

AR was supported by FWF-Project P 32905-N, DNN and GS by FWF-Project P 33417-N.

$凶 \quad$ Armin Rainer

armin.rainer@univie.ac.at

David Nicolas Nenning

david.nicolas.nenning@univie.ac.at

Gerhard Schindl

gerhard.schindl@univie.ac.at

1 Fakultät für Mathematik, Universität Wien, Oskar-Morgenstern-Platz 1, 1090 Wien, Austria 
$d \geq 1$, and on smooth manifolds. (On the other hand, the regularity of a single power of a function generally says nothing about the regularity of the function itself; e.g. $\left(\mathbf{1}_{\mathbb{Q}}-\mathbf{1}_{\mathbb{R} \backslash \mathbb{Q}}\right)^{2}=1$, where $\mathbf{1}_{A}$ is the indicator function of a set $A$.)

It was soon realized that the statement also holds for complex valued functions and it led to the study of so-called pseudoimmersions $[7,12,13,19]$. A simple proof based on ring theory was given by [1].

Only recently Thilliez [30] showed that Joris's result carries over to DenjoyCarleman classes of Roumieu type $\mathcal{E}^{\{M\}}$. These are ultradifferentiable classes of smooth functions defined by certain growth properties imposed upon the sequence of iterated derivatives in terms of a weight sequence $M$ (which in view of the Cauchy estimates measures the deviation from analyticity).

By extracting the essence of Thilliez's proof, we show in this paper that a broad variety of ultradifferentiable classes has a division property equivalent to Joris's result. Let $\mathcal{S}$ be a subring (with multiplicative identity) of the ring of germs at $0 \in \mathbb{R}^{d}$ of complex valued $C^{\infty}$-functions. We say that $\mathcal{S}$ has the division property ( $\mathscr{D}$ ) if for any function germ $f$ at $0 \in \mathbb{R}^{d}$ we have

$$
\left(j \in \mathbb{N}_{\geq 1}, f^{j}, f^{j+1} \in \mathcal{S}\right) \Longrightarrow f \in \mathcal{S} \text {. }
$$

If $\mathcal{S}$ has property $(\mathscr{D})$, then Joris's theorem holds in $\mathcal{S}$. Indeed, suppose that $p_{1}, p_{2}$ are relatively prime positive integers and $f^{p_{1}}, f^{p_{2}} \in \mathcal{S}$. All integers $j \geq p_{1} p_{2}$ can be written $j=a_{1} p_{1}+a_{2} p_{2}$ for $a_{1}, a_{2} \in \mathbb{N}$, see [11, p.270]. Hence, $f^{j} \in \mathcal{S}$ for all $j \geq p_{1} p_{2}$. Since two consecutive integers are relatively prime, also the converse holds. $\overline{1}$

\subsection{Results}

Let us give an overview of our results.

The rings of germs in one dimension $d=1$ of the following ultradifferentiable classes have property $(\mathscr{D})$ :

- $\mathcal{E}^{[M]}$, Denjoy-Carleman class of Roumieu (Theorems 2.2 and 2.3) and Beurling type (Theorem 2.6),

- $\mathcal{E}^{[\omega]}$, Braun-Meise-Taylor classes of Roumieu and Beurling type (Theorem 3.1),

- $\mathcal{E}^{[\mathfrak{M}]}$, ultradifferentiable classes defined by weight matrices of Roumieu and Beurling type (Theorem 4.2).

It is understood that certain minimal regularity properties of the weights are assumed (see Table 1) which in particular guarantee that the sets of germs are indeed rings. (Note that by convention [.] stands for $\{\cdot\}$, i.e., Roumieu, as well as (.), i.e., Beurling.)

Interestingly, the proof in one dimension works for quasianalytic and nonquasianalytic classes alike. But the tool used to reduce the multidimensional to the one-dimensional statement is only available in the non-quasianalytic Roumieu

\footnotetext{
${ }^{1}$ In an earlier version of the paper we considered the division property $\left(j \in \mathbb{N}_{\geq 1}, g, q, f g \in \mathcal{S}, f^{j}=\right.$ $q g) \Longrightarrow f \in \mathcal{S}$ which resulted from our wish to prove an ultradifferentiable version of the division theorem [13, Theorem 1]. But this property is equivalent to (2).
} 
case ([15], [27]). The (multidimensional) Beurling case can often be reduced to the corresponding Roumieu case. Hence we obtain the following multidimensional non-quasianalytic results. The rings of germs in all dimensions $d$ of the following ultradifferentiable classes have property $(\mathscr{D})$ :

- $\mathcal{E}^{[M]}$, non-quasianalytic Denjoy-Carleman classes of Roumieu (Theorem 2.2) and Beurling type (Theorem 2.5),

- $\mathcal{E}^{[\omega]}$, non-quasianalytic Braun-Meise-Taylor classes of Roumieu and Beurling type (Theorem 3.2),

- $\mathcal{E}^{\{\mathfrak{M}\}}$, non-quasianalytic ultradifferentiable classes defined by weight matrices of Roumieu type (Theorem 4.3).

For quasianalytic Denjoy-Carleman classes of Roumieu type $\mathcal{E}^{\{M\}}$ in one dimension the implication (2) follows from the stronger result, due to Thilliez [29], that $C^{\infty}$-solutions of a polynomial equation

$$
z^{n}+a_{1} z^{n-1}+\cdots+a_{n-1} z+a_{n}=0
$$

where the coefficients $a_{j}$ are germs at $0 \in \mathbb{R}$ of $\mathcal{E}^{\{M\}}$-functions, are of class $\mathcal{E}^{\{M\}}$ (under weak assumptions on $M$ ). This is false for non-quasianalytic classes. But it seems to be unknown whether, in the presence of quasianalyticity, it holds in higher dimensions. In fact, quasianalytic ultradifferentiability cannot be tested on quasianalytic curves (or lower dimensional plots) even if the function in question is known to be smooth ( $[10,20])$.

Hence, we think that it is interesting that, combining our proof with a description of certain quasianalytic classes $\mathcal{E}^{\{M\}}$ as an intersection of suitable non-quasianalytic ones (due to [16]), we obtain that these quasianalytic classes have property ( $\mathscr{D})$ in all dimensions (see Theorem 2.7 and also Remarks 3.3 and 4.4).

Since all considered regularity classes are local, the results for germs immediately give corresponding results for functions on open sets.

\subsection{Summary of the Results}

We list in Table 1 the ultradifferentiable rings of germs known to have property ( $\mathscr{D})$, together with the needed assumptions on the weights and the respective references. All germs are function germs at 0 in $\mathbb{R}^{d}$ for some dimension $d$. The dimension is added as a left subscript, e.g., ${ }_{d} \mathcal{E}^{[M]}$ denotes the ring of germs at $0 \in \mathbb{R}^{d}$ of $\mathcal{E}^{[M]}$-functions. All notions will be defined below.

The Roumieu parts of the results in the first and the fifth row are due to Thilliez [30]; see Sects. 2.4, 2.5.

We remark that non-quasianalytic Denjoy-Carleman classes $\mathcal{E}^{\{M\}}$, where the weight sequence $M$ lacks moderate growth, do not have property $(\mathscr{D})$ in general; see [30, Remark 2.2.3]. The moderate growth condition is rather restrictive (e.g., it implies that the class $\mathcal{E}^{\{M\}}$ is contained in a Gevrey class). The consideration of the classes $\mathcal{E}^{[\omega]}$ and $\mathcal{E}^{[\mathfrak{M}]}$ allows to overcome this restriction in the sense that the implication (2) holds under weaker moderate growth conditions. 
Table 1 Ultradifferentiable rings of germs having property $(\mathscr{D})$

\begin{tabular}{|c|c|c|}
\hline \multicolumn{2}{|c|}{ Quasianalytic ring of germs } & Reference \\
\hline${ }_{1} \mathcal{E}^{[M]}$ & $\begin{array}{l}\text { derivation closed } \\
m \text { log-convex } \\
m_{k}^{1 / k} \rightarrow \infty \text { (Beurling) }\end{array}$ & Theorems 2.3, 2.6 \\
\hline${ }_{1} \mathcal{E}^{[\omega]}$ & $\omega$ concave & Theorem 3.1 \\
\hline${ }_{1} \mathcal{E}^{[\mathfrak{M}]}$ & $\begin{array}{l}\text { [regular] } \\
\text { [moderate growth] }\end{array}$ & Theorem 4.2 \\
\hline${ }_{d} \mathcal{E}^{\{M\}}$ & $\begin{array}{l}\text { intersectable } \\
\text { moderate growth }\end{array}$ & Theorem 2.7 \\
\hline Non-qu & tic ring of germs & Reference \\
\hline${ }_{d} \mathcal{E}^{[M]}$ & $\begin{array}{l}\text { moderate growth } \\
m \text { log-convex }\end{array}$ & Theorems 2.2, 2.5 \\
\hline${ }_{d} \mathcal{E}^{[\omega]}$ & $\omega$ concave & Theorem 3.2 \\
\hline${ }_{1} \mathcal{E}^{[\mathfrak{M}]}$ & $\begin{array}{l}\text { [regular] } \\
\text { [moderate growth] }\end{array}$ & Theorem 4.2 \\
\hline${ }_{d} \mathcal{E}^{\{\mathfrak{M}\}}$ & $\begin{array}{l}\text { \{regular } \\
\{\text { moderate growth }\end{array}$ & Theorem 4.3 \\
\hline
\end{tabular}

\subsection{Strategy of the Proof}

Thilliez's proof of Joris's theorem for $\mathcal{E}^{\{M\}}$ consists of the following two steps:

(i) The class $\mathcal{E}^{\{M\}}$ admits a description by holomorphic approximation which is based on a result of Dynkin [8] on almost analytic extensions and a related $\bar{\partial}$-problem.

(ii) If $f^{j}, f^{j+1}$ are of class $\mathcal{E}^{\{M\}}$ and $g_{\varepsilon}, h_{\varepsilon}$ are respective holomorphic approximations, the quotient $h_{\varepsilon} / g_{\varepsilon}$ is a naive candidate for a holomorphic approximation of $f$. In order to avoid small divisors one considers

$$
u_{\varepsilon}=\varphi_{\varepsilon} \frac{\bar{g}_{\varepsilon} h_{\varepsilon}}{\max \left\{\left|g_{\varepsilon}\right|, r_{\varepsilon}\right\}^{2}},
$$

where $\varphi_{\varepsilon}$ is a suitable cutoff function and $r_{\varepsilon}>0$. For good choices of $r_{\varepsilon}$ the function $u_{\varepsilon}$ has uniform bounds and is close to $f$. The solution of a $\bar{\partial}$-problem is used to modify $u_{\varepsilon}$ in order to obtain a holomorphic approximation of $f$. By step (i) we may conclude that $f$ belongs to $\mathcal{E}^{\{M\}}$.

Following the same strategy, we will work with weight matrices $\mathfrak{M}$, since they provide a framework for ultradifferentiability (Sect. 4) which encompasses DenjoyCarleman classes (Sect. 2) and Braun-Meise-Taylor classes (Sect. 3). In Sect. 5 we prove a general characterization result by holomorphic approximation for $\mathcal{E}^{[\mathfrak{M}]}$ (Theorem 5.3) which extends step (i); it builds on the description by almost analytic extension presented in our recent paper [9]. Then we execute a version of step (ii) under a quite minimal set of assumptions, see Lemma 6.1. It enables us to easily deduce the main results in Sect. 6. 


\section{Denjoy-Carleman Classes Have Property ( $\mathscr{D})$}

\subsection{Weight Sequences and Denjoy-Carleman Classes}

Let $\mu=\left(\mu_{k}\right)$ be a positive increasing (i.e. $\mu_{k} \leq \mu_{k+1}$ ) sequence with $\mu_{0}=1$. We define a sequence $M$ by setting $M_{k}:=\mu_{1} \cdots \mu_{k}, M_{0}:=1$, and a sequence $m$ by $m_{k}:=\frac{M_{k}}{k !}$. Clearly, $\mu$ uniquely determines $M$ and $m$, and vice versa. In analogy we shall use sequences $N \leftrightarrow n \leftrightarrow \nu, L \leftrightarrow \ell \leftrightarrow \lambda$, etc.

That $\mu$ is increasing means that $M$ is $\log$-convex, i.e., $\log M$ is convex or, equivalently, $M_{k}^{2} \leq M_{k-1} M_{k+1}$ for all $k$. If in addition $M_{k}^{1 / k} \rightarrow \infty$, we say that $M$ is a weight sequence.

Sometimes we will make the stronger assumption that $m$ is log-convex.

For $\sigma>0$ and open $U \subseteq \mathbb{R}^{d}$, one defines the Banach space

$$
\mathcal{B}_{\sigma}^{M}(U):=\left\{f \in C^{\infty}(U):\|f\|_{\sigma, U}^{M}:=\sup _{x \in U, \alpha \in \mathbb{N}^{d}} \frac{\left|\partial^{\alpha} f(x)\right|}{\sigma^{|\alpha|} M_{|\alpha|}}<\infty\right\}
$$

and the (local) Denjoy-Carleman classes of Roumieu type

$$
\mathcal{E}^{\{M\}}(U):=\operatorname{proj}_{V \Subset U} \operatorname{ind}_{\sigma>0} \mathcal{B}_{\sigma}^{M}(V) .
$$

For later reference we also consider the global class $\mathcal{B}^{\{M\}}(V):=\operatorname{ind}_{\sigma>0} \mathcal{B}_{\sigma}^{M}(V)$. Replacing the existential quantifier for $\sigma$ by a universal quantifier, we find the DenjoyCarleman classes of Beurling type

$$
\mathcal{E}^{(M)}(U):=\operatorname{proj}_{V \Subset U} \operatorname{proj}_{\sigma>0} \mathcal{B}_{\sigma}^{M}(V)
$$

and $\mathcal{B}^{(M)}(V):=\operatorname{proj}_{\sigma>0} \mathcal{B}_{\sigma}^{M}(V)$. We use the notation $\mathcal{E}^{[M]}$ for both $\mathcal{E}^{\{M\}}$ and $\mathcal{E}^{(M)}$, similarly for $\mathcal{B}^{[M]}$, etc.

For positive sequences $M, N$, we write $M \preccurlyeq N$ if $\sup _{k \in \mathbb{N}}\left(\frac{M_{k}}{N_{k}}\right)^{1 / k}<\infty$ and $M \triangleleft N$ if $\lim _{k \rightarrow \infty}\left(\frac{M_{k}}{N_{k}}\right)^{1 / k}=0$. We have (cf. [23, Proposition 2.12])

$$
\begin{aligned}
M \preccurlyeq N & \Leftrightarrow \quad \mathcal{E}^{[M]}(U) \subseteq \mathcal{E}^{[N]}(U), \\
M \triangleleft N & \Leftrightarrow \quad \mathcal{E}^{\{M\}}(U) \subseteq \mathcal{E}^{(N)}(U),
\end{aligned}
$$

where for " $\Leftarrow$ " one has to assume that $M$ is a weight sequence. Note that $\mathcal{E}^{\{(k !)\}}(U)$ coincides with $C^{\omega}(U)$ and so the class of real analytic functions is contained in $\mathcal{E}^{(M)}(\subseteq$ $\left.\mathcal{E}^{\{M\}}\right)$ if and only if $m_{k}^{1 / k} \rightarrow \infty$.

Log-convexity of $M$ implies that $\mathcal{E}^{[M]}(U)$ is closed under pointwise multiplication of functions. Additional regularity properties for $M$ endow $\mathcal{E}^{[M]}(U)$ with additional structure, e.g., log-convexity of $m$ implies closedness under composition of functions. A crucial assumption in [30] is moderate growth of $M$, which reads as follows

$$
\exists C>0 \forall k, j \in \mathbb{N}: M_{k+j} \leq C^{k+j} M_{k} M_{j} .
$$


It implies derivation closedness

$$
\exists C>0 \forall k \in \mathbb{N}: M_{k+1} \leq C^{k+1} M_{k}
$$

The last property we need to mention is non-quasianalyticity of $M$, that is

$$
\sum_{k=1}^{\infty} \frac{1}{\mu_{k}}<\infty, \quad \text { or equivalently } \quad \sum_{k=1}^{\infty} \frac{1}{M_{k}^{1 / k}}<\infty
$$

By the Denjoy-Carleman theorem, this condition is equivalent to the existence of non-trivial functions with compact support in $\mathcal{E}^{[M]}(U)$. It is well-known that nonquasianalyticity implies $m_{k}^{1 / k} \rightarrow \infty$.

Let ${ }_{d} \mathcal{E}^{[M]}$ denote the ring of germs at $0 \in \mathbb{R}^{d}$ of complex valued $\mathcal{E}^{[M]}$-functions; here we assume that $M$ is a weight sequence in order to have a ring.

Remark 2.1 There is a slight mismatch between our notation (also used in [9]) and that of [30] (and [22]). We write $M_{j}=m_{j} j$ ! for weight sequences, so our $m$ corresponds to $M$ in [30].

\subsection{Associated Functions}

Let $m=\left(m_{k}\right)$ be a positive sequence with $m_{0}=1$ and $m_{k}^{1 / k} \rightarrow \infty$. We define the function

$$
h_{m}(t):=\inf _{k \in \mathbb{N}} m_{k} t^{k}, \quad \text { for } t>0, \quad \text { and } \quad h_{m}(0):=0,
$$

which is is increasing, continuous on $[0, \infty)$, and positive for $t>0$. For large $t$ we have $h_{m}(t)=1$. Furthermore, we need

$$
\bar{\Gamma}_{m}(t):=\min \left\{k: h_{m}(t)=m_{k} t^{k}\right\}, \quad t>0,
$$

and, provided that $m_{k+1} / m_{k} \rightarrow \infty$,

$$
\underline{\Gamma}_{m}(t):=\min \left\{k: \frac{m_{k+1}}{m_{k}} \geq \frac{1}{t}\right\}, \quad t>0 .
$$

We trivially have $\underline{\Gamma}_{m} \leq \bar{\Gamma}_{m}$. If $m$ is log-convex, then $\bar{\Gamma}_{m}=\underline{\Gamma}_{m}$.

We shall use these functions for $m_{k}=M_{k} / k$ !, where $M$ is a weight sequence satisfying $m_{k}^{1 / k} \rightarrow \infty$. Then $m_{k+1} / m_{k} \rightarrow \infty\left(\right.$ since $M_{k}^{1 / k} \leq \mu_{k}$ for all $\left.k\right)$.

\subsection{Regular Weight Sequences}

A weight sequence $M$ is said to be regular if $m_{k}^{1 / k} \rightarrow \infty, M$ is derivation closed, and there exists a constant $C \geq 1$ such that $\bar{\Gamma}_{m}(C t) \leq \underline{\Gamma}_{m}(t)$ for all $t>0$. 


\subsection{Denjoy-Carleman Classes of Roumieu Type Have Property ( $\mathscr{D})$}

Theorem 2.2 (Non-quasianalytic ${ }_{d} \mathcal{E}^{\{M\}}$ ) Let $M$ be a non-quasianalytic regular weight sequence of moderate growth. Then, ${ }_{d} \mathcal{E}^{\{M\}}$ has property $(\mathscr{D})$.

This is a special case of Theorem 4.3 below (cf. Sect. 4.5). It implies Thilliez's result [30, Corollary 2.2.5].

A quasianalytic one-dimensional version follows from a stronger result in [29]:

Theorem 2.3 (Quasianalytic ${ }_{1} \mathcal{E}^{\{M\}}$ ) Let $M$ be a quasianalytic derivation closed weight sequence such that $m$ is log-convex. Then ${ }_{1} \mathcal{E}^{\{M\}}$ has property $(\mathscr{D})$.

\subsection{Denjoy-Carleman Classes of Beurling Type Have Property ( $\mathscr{D})$}

Let us deduce Beurling versions of Theorems 2.2 and 2.3. We use the following lemma based on [14, Lemma 6] and [9, Lemma 7.5].

Lemma 2.4 Let $L, M$ be positive sequences satisfying $L \triangleleft M$. Suppose that $m$ is logconvex and satisfies $m_{k}^{1 / k} \rightarrow \infty$. Then there exists a weight sequence $S$ such that $s$ is log-convex, $s_{k}^{1 / k} \rightarrow \infty$, and $L \leq S \triangleleft M$. Additionally, we may assume:

(i) $S$ has moderate growth, if $M$ has moderate growth.

(ii) $S$ is derivation closed, if $M$ is derivation closed.

(iii) $S$ is non-quasianalytic, if $M$ is non-quasianalytic.

Proof Only the supplements (ii) and (iii) were not already proved in [9, Lemma 7.5].

(ii) follows from the fact that a weight sequence $M$ is derivation closed if and only if there is a constant $C \geq 1$ such that $M_{k} \leq C^{k^{2}}$ for all $k$, see [17,18]. Since $S$ is a weight sequence and $S \triangleleft M$, also $S$ is derivation closed, by this criterion.

(iii) It suffices to show that there exists a non-quasianalytic weight sequence $N$ such that $L \leq N \triangleleft M$. Then we apply the lemma to $N \triangleleft M$ and obtain a weight sequence $S$ with $N \leq S \triangleleft M$ having all desired properties.

Let us show the existence of $N$. By $L \triangleleft M$, we have $\beta_{k}:=\sup _{p \geq k}\left(\frac{L_{p}}{M_{p}}\right)^{1 / p} \searrow 0$. Applying [6, Lemme 16] (see also [28, Lemma 4.1]) to $\beta_{k}$ and $\alpha_{k}=\gamma_{k}:=\frac{1}{\mu_{k}}$, yields an increasing sequence $\delta=\left(\delta_{k}\right)$ such that

$$
\begin{aligned}
& \delta_{k} \rightarrow \infty, \\
& \delta_{k} \beta_{k} \rightarrow 0, \\
& \frac{\mu_{k}}{\delta_{k}} \text { is increasing, } \\
& \sum_{k=1}^{\infty} \frac{\delta_{k}}{\mu_{k}} \leq 8 \delta_{1} \sum_{k=1}^{\infty} \frac{1}{\mu_{k}}<\infty .
\end{aligned}
$$

Then $N_{k}:=\frac{\mu_{1} \cdots \mu_{k}}{\delta_{1} \cdots \delta_{k}}$ defines a non-quasianalytic weight sequence, by (12) and (13). (Note that $v_{k}=\frac{\mu_{k}}{\delta_{k}} \rightarrow \infty$ is equivalent to $N_{k}^{1 / k} \rightarrow \infty$.) It satisfies $N \triangleleft M$ by (10). By 
(11), there is a constant $C>0$ such that $\delta_{k}\left(\frac{L_{k}}{M_{k}}\right)^{1 / k} \leq C$ for all $k$. By the monotonicity of $\delta$, this leads to $L_{k} \leq C^{k} \frac{M_{k}}{\delta_{k}^{k}} \leq C^{k} \frac{M_{k}}{\delta_{1} \cdots \delta_{k}}=C^{k} N_{k}$. After replacing $\left(N_{k}\right)$ by $\left(C^{k} N_{k}\right)$ we have $L \leq N \triangleleft M$.

Theorem 2.5 (Non-quasianalytic ${ }_{d} \mathcal{E}^{(M)}$ ) Let $M$ be a non-quasianalytic weight sequence of moderate growth such that $m$ is log-convex. Then ${ }_{d} \mathcal{E}^{(M)}$ has property $(\mathscr{D})$.

Proof Suppose that $g:=f^{j}, h:=f^{j+1} \in{ }_{d} \mathcal{E}^{(M)}$ for some positive integer $j$. Assume that representatives of these germs are defined in the neighborhood of the closure of some bounded 0 -neighborhood $U$; we denote the representatives by the same symbols. Then, the sequence

$$
L_{k}:=\max \left\{\sup _{|\alpha|=k, x \in U}\left|g^{(\alpha)}(x)\right|, \sup _{|\alpha|=k, x \in U}\left|h^{(\alpha)}(x)\right|\right\}
$$

satisfies $L \triangleleft M$. By Lemma 2.4, there exists a weight sequence $S$ satisfying the assumptions of Theorem 2.2 and $L \leq S \triangleleft M$. Thus, $f \in{ }_{d} \mathcal{E}^{\{S\}} \subseteq{ }_{d} \mathcal{E}^{(M)}$.

Theorem 2.6 (Quasianalytic ${ }_{1} \mathcal{E}^{(M)}$ ) Let $M$ be a quasianalytic derivation closed weight sequence such that $m$ is log-convex and $m_{k}^{1 / k} \rightarrow \infty$. Then ${ }_{1} \mathcal{E}^{(M)}$ has property $(\mathscr{D})$.

Proof This follows from the proof of Theorem 2.3 in [29] (which also works in the Beurling case). Alternatively, we may infer it from Theorem 2.3 by a reduction argument based on Lemma 2.4 as in the proof of Theorem 2.5.

\subsection{A Multidimensional Quasianalytic Result}

Let $M$ be a weight sequence and consider the sequence space

$$
\Lambda^{\{M\}}:=\left\{\left(c_{k}\right) \in \mathbb{C}^{\mathbb{N}}: \exists \rho>0: \sup _{k \in \mathbb{N}} \frac{\left|c_{k}\right|}{\rho^{k} M_{k}}<\infty\right\}
$$

We call a quasianalytic weight sequence $M$ intersectable if

$$
\Lambda^{\{M\}}=\bigcap_{N \in \mathcal{L}(M)} \Lambda^{\{N\}},
$$

where $\mathcal{L}(M)$ is the collection of all non-quasianalytic weight sequences $N \geq M$ such that $n$ is log-convex. The identity (15) carries over to respective function spaces, since $\mathcal{B}^{\{M\}}(U)=\left\{f \in C^{\infty}(U):\left(\sup _{x \in U}\left\|f^{(k)}(x)\right\|_{L_{\text {sym }}^{k}}\right) \in \Lambda^{\{M\}}\right\}$, where $f^{(k)}$ denotes the $k$-th order Fréchet derivative and $\|\cdot\|_{L_{\text {sym }}^{k}}$ the operator norm.

Note that a quasianalytic intersectable weight sequence $M$ always satisfies $m_{k}^{1 / k} \rightarrow$ $\infty$; an argument is given in Remark 2.8 below. 
Theorem 2.7 (Quasianalytic ${ }_{d} \mathcal{E}^{\{M\}}$ ) Let $M$ be a quasianalytic intersectable weight sequence of moderate growth. Then ${ }_{d} \mathcal{E}^{\{M\}}$ has property $(\mathscr{D})$.

The proof of this result is given in Sect. 6 .

Remark 2.8 In [16, Theorem 1.6] (inspired by [2]) a sufficient condition for intersectability was given. Let $M$ be a quasianalytic weight sequence with $1 \leq M_{0}<M_{1}$. Consider the sequence $\check{M}$ defined by

$$
\check{M}_{k}:=M_{k} \prod_{j=1}^{k}\left(1-\frac{1}{M_{j}^{1 / j}}\right)^{k}, \quad \check{M}_{0}:=1
$$

If $\check{m}$ is log-convex, then $M$ is intersectable.

Not every quasianalytic weight sequence is intersectable, for instance,

$$
\Lambda^{\{(k !)\}} \neq \bigcap_{N \in \mathcal{L}((k !))} \Lambda^{\{N\}}=\Lambda^{\{Q\}}, \quad \text { where } Q_{k}=\left(k \log (k+e)^{k} ;\right.
$$

see [16, Theorem 1.8] and [26]. Every quasianalytic intersectable weight sequence $M$ must satisfy $\Lambda^{\{Q\}} \subseteq \Lambda^{\{M\}}$, and so $m_{k}^{1 / k}$ tends to $\infty$ since clearly $q_{k}^{1 / k}$ does.

A countable family $\mathbf{Q}=\left\{Q^{n}\right\}_{n \in \mathbb{N}_{\geq 1}}$ of quasianalytic intersectable weight sequences of moderate growth was constructed in [16, Theorem 1.9]:

$$
Q_{k}^{n}=\left(k \log (k) \log (\log (k)) \cdots \log ^{[n]}(k)\right)^{k}, \quad \text { for } k \geq \exp ^{[n]}(1),
$$

where $\log ^{[n]}$ denotes the $n$-fold composition of log; analogously for $\exp ^{[n]}$.

See also [27, Sect. 11] for a generalization of this concept.

\section{Braun-Meise-Taylor Classes Have Property ( $\mathscr{D})$}

\subsection{Weight Functions and Braun-Meise-Taylor Classes}

A weight function is, by definition, a continuous increasing function $\omega:[0, \infty) \rightarrow$ $[0, \infty)$ such that

$\left(\omega_{1}\right) \omega(2 t)=O(\omega(t))$ as $t \rightarrow \infty$,

$\left(\omega_{2}\right) \omega(t)=o(t)$ as $t \rightarrow \infty$,

$\left(\omega_{3}\right) \log (t)=o(\omega(t))$ as $t \rightarrow \infty$,

$\left(\omega_{4}\right) t \mapsto \omega\left(e^{t}\right)=: \varphi_{\omega}(t)$ is convex on $[0, \infty)$.

One may assume that $\left.\omega\right|_{[0,1]} \equiv 0$ (without changing the associated classes $\mathcal{E}^{[\omega]}$ ) which we shall tacitly do if convenient.

Let $U \subseteq \mathbb{R}^{d}$ be open and $\rho>0$. We associate the Banach space

$$
\mathcal{B}_{\rho}^{\omega}(U):=\left\{f \in C^{\infty}(U):\|f\|_{\rho, U}^{\omega}:=\sup _{x \in U, \alpha \in \mathbb{N}^{d}} \frac{\left|\partial^{\alpha} f(x)\right|}{e^{\varphi_{\omega}^{*}(\rho|\alpha|) / \rho}}<\infty\right\},
$$


where $\varphi_{\omega}^{*}(s):=\sup _{t>0}\left\{s t-\varphi_{\omega}(t)\right\}$ is the Young conjugate of $\varphi_{\omega}$ (which is finite by $\left(\omega_{3}\right)$ ). Then the (local) Braun-Meise-Taylor class of Roumieu type is

$$
\mathcal{E}^{\{\omega\}}(U):=\operatorname{proj}_{V \Subset U} \operatorname{ind}_{n \in \mathbb{N}} \mathcal{B}_{n}^{\omega}(V),
$$

and that of Beurling type is

$$
\mathcal{E}^{(\omega)}(U):=\operatorname{proj}_{V \Subset U} \operatorname{proj}_{n \in \mathbb{N}} \mathcal{B}_{\frac{1}{n}}^{\omega}(V) .
$$

Again, we use $\mathcal{E}^{[\omega]}$ for $\mathcal{E}^{\{\omega\}}$ and $\mathcal{E}^{(\omega)}$, similarly for $\mathcal{B}^{[\omega]}$ etc.

For two weight functions $\omega, \sigma$ we have (cf. [23, Corollary 5.17])

$$
\begin{array}{ccc}
\sigma(t)=O(\omega(t)) \text { as } t \rightarrow \infty & \Leftrightarrow & \mathcal{E}^{[\omega]}(U) \subseteq \mathcal{E}^{[\sigma]}(U), \\
\sigma(t)=o(\omega(t)) \text { as } t \rightarrow \infty & \Leftrightarrow & \mathcal{E}^{\{\omega\}}(U) \subseteq \mathcal{E}^{(\sigma)}(U)
\end{array}
$$

We say that $\omega$ and $\sigma$ are equivalent if they generate the same classes, i.e., $\sigma(t)=$ $O(\omega(t))$ and $\omega(t)=O(\sigma(t))$ as $t \rightarrow \infty$.

A weight function is said to be non-quasianalytic if

$$
\int_{1}^{\infty} \frac{\omega(t)}{t^{2}} d t<\infty
$$

This is the case if and only if $\mathcal{E}^{[\omega]}(U)$ contains non-trivial functions of compact support (cf. [5] or [22]).

Let us emphasize that in this paper we treat condition $\left(\omega_{2}\right)$ as a general assumption for weight functions; it means that the Beurling class $\mathcal{E}^{(\omega)}$ contains the real analytic class. It is automatically satisfied if $\omega$ is non-quasianalytic.

Let ${ }_{d} \mathcal{E}^{[\omega]}$ denote the ring of germs at $0 \in \mathbb{R}^{d}$ of complex valued $\mathcal{E}^{[\omega]}$-functions; note that $\mathcal{E}^{[\omega]}$ is stable by multiplication of functions for any weight function $\omega$.

\subsection{The Associated Weight Matrix}

Let $\omega$ be a weight function. Setting $\Omega_{k}^{x}:=e^{\varphi_{\omega}^{*}(x k) / x}$ defines a weight sequence $\Omega^{x}$ for every $x>0$, where $\Omega^{x} \leq \Omega^{y}$ if $x \leq y$. Thus the collection $\Omega:=\left\{\Omega^{x}\right\}_{x>0}$ is a weight matrix (in the sense of Sect. 4). Note that $\boldsymbol{\Omega}$ satisfies a mixed moderate growth property, namely

$$
\forall x>0 \forall j, k \in \mathbb{N}: \Omega_{j+k}^{x} \leq \Omega_{j}^{2 x} \Omega_{k}^{2 x} .
$$

The importance of the associated weight matrix $\boldsymbol{\Omega}$ is that it encodes an equivalent topological description of the spaces $\mathcal{E}^{[\omega]}(U)$ as unions or intersections of DenjoyCarleman classes; see Sect. 4.5. All this can be found in [22]. 


\subsection{Braun-Meise-Taylor Classes Have Property $(\mathscr{D})$}

Theorem $3.1\left({ }_{1} \mathcal{E}^{[\omega]}\right)$ Let $\omega$ be a concave weight function. Then ${ }_{1} \mathcal{E}^{[\omega]}$ has property $(\mathscr{D})$.

Evidently, it suffices to assume that $\omega$ is equivalent to a concave weight function. For the multidimensional analogue we additionally assume non-quasianalyticity.

Theorem 3.2 (Non-quasianalytic ${ }_{d} \mathcal{E}^{[\omega]}$ ) Let $\omega$ be a non-quasianalytic concave weight function. Then ${ }_{d} \mathcal{E}^{[\omega]}$ has property $(\mathscr{D})$.

Theorems 3.1, 3.2 are corollaries of Theorems 4.2 and 4.3 below; for the proofs see Sect. 6.

Remark 3.3 Every weight sequence $M$ in the family $\mathbf{Q}$ mentioned at the end of Remark 2.8 satisfies

$$
\liminf _{k \rightarrow \infty} \frac{\mu_{a k}}{\mu_{k}}>1
$$

for some positive integer $a$. Hence there is a quasianalytic weight function $\omega_{M}$ (take, e.g., $\left.\omega_{M}(t):=-\log h_{M}(1 / t)\right)$ such that ${ }_{d} \mathcal{E}^{\left[\omega_{M}\right]}={ }_{d} \mathcal{E}^{[M]}$, by [4, Theorem 14]. So for all $M \in \mathbf{Q}$, the quasianalytic ring ${ }_{d} \mathcal{E}^{\left\{\omega_{M}\right\}}$ has property $(\mathscr{D})$.

\section{The Most General Version of the Theorem}

Let us formulate the main theorems in the most general setting available. The conditions we put on abstract weight matrices are tailored in such a way that weight matrices associated with weight functions are contained as special classes.

\subsection{Weight Matrices and Ultradifferentiable Classes}

A weight matrix $\mathfrak{M}$ is, by definition, a family of weight sequences which is totally ordered with respect to the pointwise order relation on sequences, i.e.,

- $\mathfrak{M} \subseteq \mathbb{R}^{\mathbb{N}}$,

- each $M \in \mathfrak{M}$ is a weight sequence in the sense of Sect. 2.1,

- for all $M, N \in \mathfrak{M}$ we have $M \leq N$ or $M \geq N$.

Let $U \subseteq \mathbb{R}^{d}$ be open. Given a weight matrix $\mathfrak{M}$, we define global classes

$$
\begin{aligned}
& \mathcal{B}^{\{\mathfrak{M}\}}(U):=\operatorname{ind}_{M \in \mathfrak{M}} \mathcal{B}^{\{M\}}(U), \\
& \mathcal{B}^{(\mathfrak{M})}(U):=\operatorname{proj}_{M \in \mathfrak{M}} \mathcal{B}^{(M)}(U) .
\end{aligned}
$$

The limits in (18) and (19) can always be assumed countable, as is shown in [9, Lemma 2.5]. Writing [.] for $\{\cdot\}$ and $(\cdot)$, the local classes are defined by

$$
\mathcal{E}^{[\mathfrak{M}]}(U):=\operatorname{proj}_{V \Subset U} \mathcal{B}^{[\mathfrak{M}]}(V) .
$$


Let ${ }_{d} \mathcal{E}^{[\mathfrak{M}]}$ denote the ring of germs at $0 \in \mathbb{R}^{d}$ of complex valued $\mathcal{E}^{[\mathfrak{M}]}$-functions; notice that $\mathcal{E}^{[\mathfrak{M}]}$ is stable by multiplication of functions, since each $M \in \mathfrak{M}$ is a weight sequence.

\subsection{Regular Weight Matrices}

A weight matrix $\mathfrak{M}$ satisfying

- $m_{k}^{1 / k} \rightarrow \infty$ for all $M \in \mathfrak{M}$

is called \{regular\} or $R$-regular (for Roumieu) if

- $\forall M \in \mathfrak{M} \exists N \in \mathfrak{M} \exists C \geq 1 \forall j \in \mathbb{N}: M_{j+1} \leq C^{j+1} N_{j}$,

- $\forall M \in \mathfrak{M} \exists N \in \mathfrak{M} \exists C \geq 1 \forall t>0: \bar{\Gamma}_{n}(C t) \leq \underline{\Gamma}_{m}(t)$,

and (regular) or B-regular (for Beurling) if

- $\forall M \in \mathfrak{M} \exists N \in \mathfrak{M} \exists C \geq 1 \forall j \in \mathbb{N}: N_{j+1} \leq C^{j} M_{j}$,

- $\forall M \in \mathfrak{M} \exists N \in \mathfrak{M} \exists C \geq 1 \forall t>0: \bar{\Gamma}_{m}(C t) \leq \underline{\Gamma}_{n}(t)$.

Moreover, $\mathfrak{M}$ is called regular if it is both $\mathrm{R}$ - and B-regular. By our convention, [regular] stands for \{regular\} (i.e. R-regular) in the Roumieu case and (regular) (i.e. B-regular) in the Beurling case.

\subsection{Almost Analytic Extensions}

Let $h:(0, \infty) \rightarrow(0,1]$ be an increasing continuous function which tends to 0 as $t \rightarrow 0$. Let $\rho>0$ and let $U \subseteq \mathbb{R}^{d}$ be a bounded open set. We say that a function $f$ : $U \rightarrow \mathbb{C}$ admits an $(h, \rho)$-almost analytic extension if there is a function $F \in C_{c}^{1}\left(\mathbb{C}^{d}\right)$ and a constant $C \geq 1$ such that $\left.F\right|_{U}=f$ and

$$
|\bar{\partial} F(z)| \leq C h(\rho d(z, \bar{U})), \quad \text { for } z \in \mathbb{C}^{d},
$$

where $\bar{\partial} F(z):=\sum_{j=1}^{d} \frac{\partial F(z)}{\partial \bar{z}_{j}} d \bar{z}_{j}$ and $d(z, \bar{U}):=\inf _{x \in \bar{U}}|z-x|$ denotes the distance of $z$ to $\bar{U}$.

Let us apply this definition to the functions $h_{m}$ from (7), where $m_{k}=M_{k} / k$ ! and $M$ belongs to a given weight matrix $\mathfrak{M}$. Let $f: U \rightarrow \mathbb{C}$ be a function.

- $f$ is called $\{\mathfrak{M}\}$-almost analytically extendable if it has an $\left(h_{m}, \rho\right)$-almost analytic extension for some $M \in \mathfrak{M}$ and some $\rho>0$.

- $f$ is called $(\mathfrak{M})$-almost analytically extendable if, for all $M \in \mathfrak{M}$ and all $\rho>0$, there is an $\left(h_{m}, \rho\right)$-almost analytic extension of $f$.

Theorem 4.1 ([9, Corollaries 3.3, 3.5]) Let $\mathfrak{M}$ be a [regular] weight matrix. Let $U \subseteq \mathbb{R}^{d}$ be open. Then $f \in \mathcal{E}^{[\mathfrak{M}]}(U)$ if and only if $\left.f\right|_{V}$ is $[\mathfrak{M}]$-almost analytically extendable for each quasiconvex domain $V$ relatively compact in $U$.

In Sect. 5 we shall use [9, Proposition 3.12], which is a key ingredient of the proof of Theorem 4.1. 


\subsection{Weight Matrices of Moderate Growth}

For positive sequences $M, N$ set

$$
\operatorname{mg}(M, N):=\sup _{j, k \geq 0, j+k \geq 1}\left(\frac{M_{j+k}}{N_{j} N_{k}}\right)^{1 /(j+k)} \in(0, \infty]
$$

We say that a weight matrix $\mathfrak{M}$ has $R$-moderate growth or \{moderate growth if

$$
\forall M \in \mathfrak{M} \exists N \in \mathfrak{M}: \operatorname{mg}(M, N)<\infty
$$

and B-moderate growth or (moderate growth) if

$$
\forall M \in \mathfrak{M} \exists N \in \mathfrak{M}: \operatorname{mg}(N, M)<\infty
$$

Again we say that $\mathfrak{M}$ has moderate growth if it has $\mathrm{R}$ - and $\mathrm{B}$-moderate growth, and [moderate growth] stands for \{moderate growth\} and (moderate growth), respectively.

\subsection{Denjoy-Carleman and Braun-Meise-Taylor classes in this Framework}

By definition, Denjoy-Carleman classes are described by weight matrices $\mathfrak{M}=\{M\}$ consisting of a single weight sequence $M$. Observe that the weight matrix $\mathfrak{M}=\{M\}$ is regular if and only if the weight sequence $M$ is regular, and it has moderate growth if and only if $M$ has moderate growth.

Let $\omega$ be a weight function and let $\Omega$ be the associated weight matrix (cf. Sect. 3.2). Then, by [22, Corollaries 5.8 and 5.15], as locally convex spaces

$$
\mathcal{E}^{[\omega]}(U)=\mathcal{E}^{[\Omega]}(U)
$$

and $\mathcal{E}^{[\omega]}(U)=\mathcal{E}^{[\Omega]}(U)$ for all $\Omega \in \Omega$ if and only if

$$
\exists H \geq 1 \forall t \geq 0: 2 \omega(t) \leq \omega(H t)+H
$$

which is in turn equivalent to the fact that some (equivalently each) $\Omega \in \boldsymbol{\Omega}$ has moderate growth; see also [4].

The associated weight matrix $\boldsymbol{\Omega}$ always has moderate growth, by (17). It is equivalent to a regular weight matrix $\mathfrak{S}$ (that means $\mathcal{E}^{[\omega]}=\mathcal{E}^{[\mathfrak{S}]}$ ) if and only if $\omega$ is equivalent to a concave weight function. In fact, a $\mathcal{E}^{[\omega]}$-version of the almost analytic extension theorem 4.1 holds if and only if $\omega$ is equivalent to a concave weight function; see [9, Theorem 4.8]. The weight matrix $\mathfrak{S}=\left\{S^{x}\right\}_{x>0}$ has the property that for each $x>0$ the sequence $s^{x}$ is $\log$-convex and satisfies $\operatorname{mg}\left(s^{x}, s^{2 x}\right)=: H<\infty$ and thus $h_{s^{x}}(t) \leq h_{s^{2 x}}(H t)^{2}$ for all $x, t>0$; see [25, Proposition 3]. 


\subsection{General Ultradifferentiable classes Have Property $(\mathscr{D})$}

Theorem $4.2\left({ }_{1} \mathcal{E}^{[\mathfrak{M}]}\right)$ Let $\mathfrak{M}$ be a [regular] weight matrix of [moderate growth]. Then ${ }_{1} \mathcal{E}^{[\mathfrak{M}]}$ has property $(\mathscr{D})$.

The proof is given in Sect. 6. It builds upon a characterization of the class $\mathcal{E}^{[\mathfrak{M}]}$ by holomorphic approximation; see Sect. 5.

We may infer a multidimensional result, since non-quasianalytic $\mathcal{E}^{\{\mathfrak{M}\}}$-regularity can be tested along curves; this useful tool is available in a satisfactory manner only in the non-quasianalytic Roumieu setting. We need two additional properties of the weight matrix:

$$
\exists M \in \mathfrak{M}: \sum_{k=0}^{\infty} \frac{1}{\mu_{k}}<\infty
$$

which means that $\mathcal{E}^{\{\mathfrak{M}\}}$ admits non-trivial functions of compact support, and

$$
\forall M \in \mathfrak{M} \exists N \in \mathfrak{M}: m^{\circ} \preccurlyeq n
$$

where $m_{k}^{\circ}:=\max \left\{m_{j} m_{\alpha_{1}} \cdots m_{\alpha_{j}}: \alpha_{i} \in \mathbb{N}_{>0}, \alpha_{1}+\cdots+\alpha_{j}=k\right\}$. Condition (24) is equivalent to composition closedness of $\mathcal{E}^{\{\mathfrak{M}\}}$ (which follows from the arguments in [22, Theorem 4.9]) and is satisfied by every R-regular weight matrix. Indeed, if $\mathfrak{M}$ is R-regular, then $\mathcal{E}^{\{\mathfrak{M}\}}$ has a description by almost analytic extension, by Theorem 4.1. It is easy to see (cf. [9, Proposition 1.1]) that the latter condition is preserved by composition of functions.

Under these assumptions, a function $f$ defined on an open set $U \subseteq \mathbb{R}^{d}$ is of class $\mathcal{E}^{\{\mathfrak{M}\}}$ if and only if $f \circ c$ is of class $\mathcal{E}^{\{\mathfrak{M}\}}$ for all $\mathcal{E}^{\{\mathfrak{M}\}}$-curves in $U$; see $[15,16]$ and [27, Theorem 10.7.1].

Theorem 4.3 (Non-quasianalytic ${ }_{d} \mathcal{E}^{\{\mathfrak{M}\}}$ ) Let $\mathfrak{M}$ be an R-regular weight matrix of $R$-moderate growth satisfying (23). Then ${ }_{d} \mathcal{E}^{\{\mathfrak{M}\}}$ has property $(\mathscr{D})$.

Proof This follows immediately from Theorem 4.2 and the above observations.

Note that Theorem 4.3 implies Theorem 2.2 as a special case.

Remark 4.4 The family $\mathbf{Q}=\left\{Q^{n}\right\}_{n \in \mathbb{N}_{\geq 1}}$ of quasianalytic intersectable weight sequences referred to at the end of Remark 2.8 actually is a regular weight matrix of moderate growth. The Roumieu class $\mathcal{E}^{\{\mathbf{Q}\}}$ is quasianalytic and, since Theorem 2.7 applies to every $M \in \mathbf{Q}$, we conclude that ${ }_{d} \mathcal{E}^{\{\mathbf{Q}\}}$ has property $(\mathscr{D})$.

Note that there is no weight sequence $M$ with $\mathcal{E}^{\{M\}}=\mathcal{E}^{\{\mathbf{Q}\}}$ and no weight function $\omega$ with $\mathcal{E}^{\{\omega\}}=\mathcal{E}^{\{\mathbf{Q}\}}$. This follows from the fact that $Q^{n} \leq Q^{n+1} \npreceq Q^{n}$, in analogy to the proof given in [22, Theorem 5.22]; see also Remark 5.25 there. 


\section{Holomorphic Approximation of Functions in $\mathcal{E}^{[\mathfrak{M}]}$}

In this section we prove a characterization of the class $\mathcal{E}^{[\mathfrak{M}]}$ (in dimension one) by holomorphic approximation. It generalizes [30, Proposition 3.3.2].

For notational convenience, we set $\|f\|_{A}:=\sup _{z \in A}|f(z)|$ for any complex valued function $f$, where $A$ is any set in the domain of $f$.

\subsection{Some Preparatory Observations}

Lemma 5.1 Let $M, N$ be weight sequences satisfying $m_{k}^{1 / k} \rightarrow \infty, n_{k}^{1 / k} \rightarrow \infty$, and $C:=\operatorname{mg}(M, N)<\infty$. Then

$$
\begin{aligned}
& h_{m}(t) \leq C^{j} n_{j} t^{j} h_{n}(C t), \quad t>0, j \in \mathbb{N}, \\
& h_{m}(t) \leq h_{n}\left(\frac{e C}{2} t\right)^{2}, \quad t>0 .
\end{aligned}
$$

Proof Note that $\operatorname{mg}(m, n) \leq \operatorname{mg}(M, N)$. Thus, for all $j \in \mathbb{N}$ and $t>0$,

$$
h_{m}(t) \leq \inf _{k \geq 0} m_{k+j} t^{k+j} \leq \inf _{k \geq 0} n_{j} n_{k}(C t)^{k+j}=C^{j} n_{j} t^{j} h_{n}(C t) .
$$

For (26) we refer to [24, Lemma 3.13].

For $\varepsilon>0$ let $\Omega_{\varepsilon}$ denote the interior of the ellipse in $\mathbb{C}$ with vertices $\pm \cosh (\varepsilon)$ and co-vertices $\pm i \sinh (\varepsilon)$. By $\mathcal{H}\left(\Omega_{\varepsilon}\right)$ we denote the space of holomorphic functions on $\Omega_{\varepsilon}$. The following lemma is a simple modification of [30, Lemma 3.2.4].

Lemma 5.2 Let $M, N$ be two weight sequences satisfying $m_{k}^{1 / k} \rightarrow \infty, n_{k}^{1 / k} \rightarrow \infty$, and $C:=\operatorname{mg}(M, N)<\infty$. Let $\varepsilon>0$. Let $g \in \mathcal{H}\left(\Omega_{\varepsilon}\right) \cap C^{0}\left(\bar{\Omega}_{\varepsilon}\right)$ and assume that there are constants $L, a_{1}, a_{2}>0$ such that

$$
\|g\|_{\Omega_{\varepsilon}} \leq L, \quad\|g\|_{[-1,1]} \leq a_{1} h_{m}\left(a_{2} \varepsilon\right) .
$$

Then with $a_{3}:=\max \left\{a_{1}, L\right\}$ and $a_{4}:=e C a_{2}$ we have

$$
\|g\|_{\Omega_{\varepsilon / 2}} \leq a_{3} h_{n}\left(a_{4} \varepsilon\right)
$$

Proof Let $f(z):=\frac{1}{a_{1}} g(\sin (\varepsilon z))$. Since $z \mapsto \sin (\varepsilon z)$ maps the horizontal strip $S:=$ $\{z \in \mathbb{C}:|\operatorname{Im}(z)|<1\}$ to $\Omega_{\varepsilon}$, we get that $f \in \mathcal{H}(S) \cap C^{0}(\bar{S})$ is bounded by $K:=\max \left\{1, \frac{L}{a_{1}}\right\}$ on the whole of $S$ and by $h_{m}\left(a_{2} \varepsilon\right)$ on $\mathbb{R}$. Thus an application of Hadamard's three lines theorem gives

$$
|f(z)| \leq h_{m}\left(a_{2} \varepsilon\right)^{1-|\operatorname{Im}(z)|} K^{|\operatorname{Im}(z)|}, \quad z \in S .
$$


Since $h_{m} \leq 1$ and every $w \in \Omega_{\varepsilon / 2}$ can be written as $w=\sin (\varepsilon z)$ for some $w \in S$ with $|\operatorname{Im}(w)| \leq 1 / 2$, we obtain

$$
|g(w)| \leq a_{1}\left(K h_{m}\left(a_{2} \varepsilon\right)\right)^{1 / 2}
$$

The statement follows from (26).

\subsection{Condition $\left(\mathcal{P}_{[\mathfrak{M}]}\right)$}

Let $\mathfrak{M}$ be a weight matrix.

$\left(\mathcal{P}_{\{\mathfrak{M}\}}\right)$ We say that a function $f:[-1,1] \rightarrow \mathbb{C}$ satisfies $\left(\mathcal{P}_{\{\mathfrak{M}\}}\right)$ if there exist $M \in \mathfrak{M}$, constants $K, c_{1}, c_{2}>0$, and a family $\left(f_{\varepsilon}\right)_{0<\varepsilon \leq \varepsilon_{0}}$ of functions $f_{\varepsilon} \in \mathcal{H}\left(\Omega_{\varepsilon}\right) \cap C^{0}\left(\bar{\Omega}_{\varepsilon}\right)$ such that for all $0<\varepsilon \leq \varepsilon_{0}$,

$$
\begin{aligned}
\left\|f_{\varepsilon}\right\|_{\Omega_{\varepsilon}} & \leq K, \\
\left\|f-f_{\varepsilon}\right\|_{[-1,1]} & \leq c_{1} h_{m}\left(c_{2} \varepsilon\right) .
\end{aligned}
$$

$\left(\mathcal{P}_{(\mathfrak{M})}\right)$ We say that a function $f:[-1,1] \rightarrow \mathbb{C}$ satisfies $\left(\mathcal{P}_{(\mathfrak{M})}\right)$ if for all $M \in \mathfrak{M}$ and all $c_{2}>0$ there exist constants $K, c_{1}>0$ and a family $\left(f_{\varepsilon}\right)_{0<\varepsilon<\varepsilon_{0}}$ of functions $f_{\varepsilon} \in \mathcal{H}\left(\Omega_{\varepsilon}\right) \cap C^{0}\left(\bar{\Omega}_{\varepsilon}\right)$ such that (27) and (28) hold for all $0<\varepsilon \leq \varepsilon_{0}$.

Note that $\left(\mathcal{P}_{\{\mathfrak{M}\}}\right)$ generalizes condition $\left(\mathcal{P}_{M}\right)$ of [30].

\subsection{Description by Holomorphic Approximation}

Theorem 5.3 (i) Let $M^{(i)}, 1 \leq i \leq 3$, be weight sequences with $\left(m_{k}^{(i)}\right)^{1 / k} \rightarrow \infty$ and

$$
\begin{aligned}
& \exists B_{1} \geq 1 \forall t>0: \bar{\Gamma}_{m^{(2)}}\left(B_{1} t\right) \leq \Gamma_{m^{(1)}}(t), \\
& \exists B_{2} \geq 1 \forall j \in \mathbb{N}: m_{j+1}^{(2)} \leq B_{2}^{j+1} m_{j}^{(3)} .
\end{aligned}
$$

Then for each $f \in \mathcal{B}_{B_{0}}^{M^{(1)}}((-1,1))$ there exist positive constants $K, c_{1}, c_{2}$ and functions $f_{\varepsilon} \in \mathcal{H}\left(\Omega_{\varepsilon}\right) \cap C^{0}\left(\bar{\Omega}_{\varepsilon}\right)$ such that for all small $\varepsilon>0$

$$
\left\|f_{\varepsilon}\right\|_{\Omega_{\varepsilon}} \leq K, \quad\left\|f-f_{\varepsilon}\right\|_{[-1,1]} \leq c_{1} h_{m^{(3)}}\left(c_{2} \varepsilon\right) .
$$

The constants $K, c_{1}, c_{2}$ only depend on $B_{i}$, in particular, $c_{2}=C B_{0} B_{1}$, where $C$ is an absolute constant.

(ii) Let $N^{(i)}, 1 \leq i \leq 3$, be weight sequences with $\left(n_{k}^{(i)}\right)^{1 / k} \rightarrow \infty$ and $m g\left(N^{(i)}, N^{(i+1)}\right)=D^{(i)}<\infty$. Let $f:[-1,1] \rightarrow \mathbb{C}$ be a function. Assume that there exist positive constants $K, c_{1}, c_{2}$ and functions $f_{\varepsilon} \in \mathcal{H}\left(\Omega_{\varepsilon}\right) \cap C^{0}\left(\bar{\Omega}_{\varepsilon}\right)$ such that for all small $\varepsilon>0$

$$
\left\|f_{\varepsilon}\right\|_{\Omega_{\varepsilon}} \leq K, \quad\left\|f-f_{\varepsilon}\right\|_{[-1,1]} \leq c_{1} h_{n^{(1)}}\left(c_{2} \varepsilon\right)
$$


Then $f \in \mathcal{B}_{\sigma}^{N^{(3)}}((-b, b))$ for every $b<1$, where $\sigma:=\frac{2 e D^{(1)} D^{(2)} c_{2}}{E(1-b)}$ and $E$ is an absolute constant.

(iii) If $\mathfrak{M}$ is a [regular] weight matrix of [moderate growth], then

$$
f \in \mathcal{B}^{[\mathfrak{M}]}((-1,1)) \Rightarrow f \text { satisfies }\left(\mathcal{P}_{[\mathfrak{M}]}\right) \Rightarrow f \in \mathcal{E}^{[\mathfrak{M}]}((-1,1)) .
$$

Note that [regularity] of $\mathfrak{M}$ is needed for the first implication in (iii), [moderate growth] for the second. Item (iii) generalizes [30, Proposition 3.3.2].

Proof We follow closely the proof of [30, Proposition 3.3.2].

(i) Let $f \in \mathcal{B}_{B_{0}}^{M^{(1)}}((-1,1))$. By [9, Proposition 3.12], there are constants $c_{1}, c_{2}>0$ and a function $F \in C_{c}^{1}(\mathbb{C})$ extending $f$ such that

$$
|\bar{\partial} F(z)| \leq c_{1} h_{m^{(3)}}\left(c_{2} d(z,[-1,1])\right), \quad z \in \mathbb{C} .
$$

Note that $c_{1}=c_{1}\left(\|f\|_{B_{0}}^{M^{(1)}}, B_{0}, B_{1}, B_{2}\right)$ and $c_{2}=12 B_{0} B_{1}$. Then $w_{\varepsilon}:=\bar{\partial} F \mathbf{1}_{\Omega_{\varepsilon}}$ satisfies

$$
\left\|w_{\varepsilon}\right\|_{\mathbb{C}} \leq c_{1} h_{m^{(3)}}\left(C c_{2} \varepsilon\right)
$$

where $C>0$ is an absolute constant such that $d(z,[-1,1]) \leq C \varepsilon$ for $z \in \Omega_{\varepsilon}$. Moreover, the bounded continuous function

$$
v_{\varepsilon}(z):=\frac{1}{2 \pi i} \int_{\mathbb{C}} \frac{w_{\varepsilon}(\zeta)}{\zeta-z} d \zeta \wedge d \bar{\zeta}
$$

satisfies $\bar{\partial} v_{\varepsilon}=w_{\varepsilon}$ in the distributional sense, and we have

$$
\left\|v_{\varepsilon}\right\|_{\mathbb{C}} \leq c_{1} h_{m^{(3)}}\left(C c_{2} \varepsilon\right)
$$

So $f_{\varepsilon}:=F-v_{\varepsilon}$ is holomorphic on $\Omega_{\varepsilon}$ and continuous on $\bar{\Omega}_{\varepsilon}$. The estimates (34) and (35) easily imply (31).

(ii) Let $f:[-1,1] \rightarrow \mathbb{C}$ satisfy (32). Consider $g_{\varepsilon}:=f_{\varepsilon}-f_{2 \varepsilon} \in \mathcal{H}\left(\Omega_{\varepsilon}\right) \cap C^{0}\left(\bar{\Omega}_{\varepsilon}\right)$. Then $\left\|g_{\varepsilon}\right\|_{\Omega_{\varepsilon}} \leq 2 K$ and $\left\|g_{\varepsilon}\right\|_{[-1,1]} \leq 2 c_{1} h_{n^{(1)}}\left(2 c_{2} \varepsilon\right)$. By Lemma 5.2,

$$
\left\|g_{\varepsilon}\right\|_{\Omega_{\varepsilon / 2}} \leq \max \left\{c_{1}, 2 K\right\} h_{n^{(2)}}\left(2 e D^{(1)} c_{2} \varepsilon\right) .
$$

There exists a (universal) constant $E>0$ such that for any $b<1$ the closed disk with radius $E(1-b) \varepsilon$ around any $x \in[-b, b]$ is contained in $\Omega_{\varepsilon / 2}$. The Cauchy estimates and (25) yield

$$
\begin{aligned}
\left\|g_{\varepsilon}^{(j)}\right\|_{[-b, b]} & \leq \frac{\max \left\{c_{1}, 2 K\right\}}{(E(1-b) \varepsilon)^{j}} j ! h_{n^{(2)}}\left(2 e D^{(1)} c_{2} \varepsilon\right) \\
& \leq \max \left\{c_{1}, 2 K\right\}\left(\frac{2 e D^{(1)} D^{(2)} c_{2}}{E(1-b)}\right)^{j} N_{j}^{(3)} h_{n^{(3)}}\left(2 e D^{(1)} D^{(2)} c_{2} \varepsilon\right),
\end{aligned}
$$


which means $\left\|g_{\varepsilon}\right\|_{\sigma,[-b, b]}^{N^{(3)}} \leq \max \left\{c_{1}, 2 K\right\} h_{n^{(3)}}\left(2 e D^{(1)} D^{(2)} c_{2} \varepsilon\right)$ for $\sigma=\frac{2 e D^{(1)} D^{(2)} c_{2}}{E(1-b)}$. Thus, if $\varepsilon_{0}>0$ is such that (32) holds for all $0<\varepsilon \leq \varepsilon_{0}$, then

$$
g:=f_{\varepsilon_{0}}+\sum_{j=1}^{\infty} g_{\varepsilon_{0} 2^{-j}}=f_{\varepsilon_{0}}+\sum_{j=1}^{\infty}\left(f_{\varepsilon_{0} 2^{-j}}-f_{\varepsilon_{0} 2^{-j+1}}\right)
$$

converges absolutely in the Banach space $\mathcal{B}_{\sigma}^{N^{(3)}}([-b, b])$. Clearly, for every $k \in \mathbb{N}$,

$$
g=f_{\varepsilon_{0} 2^{-k}}+\sum_{j=k+1}^{\infty}\left(f_{\varepsilon_{0} 2^{-j}}-f_{\varepsilon_{0} 2^{-j+1}}\right)
$$

and $f=g$ on $[-b, b]$, since for $x \in[-b, b]$,

$$
|f(x)-g(x)| \leq\left|f(x)-f_{\varepsilon_{0} 2^{-k}}(x)\right|+\left|\sum_{j=k+1}^{\infty}\left(f_{\varepsilon_{0} 2^{-j}}(x)-f_{\varepsilon_{0} 2^{-j+1}}(x)\right)\right|
$$

which tends to 0 as $k \rightarrow \infty$, by (32) and absolute convergence of the sum.

(iii) For the first implication in (33) in the Roumieu case, observe that for $f \in$ $\mathcal{B}^{\{\mathfrak{M}\}}((-1,1))$ we have $f \in \mathcal{B}_{B_{0}}^{M^{(1)}}((-1,1))$ for some $B_{0}>0$ and $M^{(1)} \in \mathfrak{M}$. Then R-regularity of $\mathfrak{M}$ implies the existence of $M^{(2)}, M^{(3)} \in \mathfrak{M}$ such that (29) and (30) are satisfied. Thus (i) yields the desired holomorphic approximation.

In the Beurling case take any weight sequence $M^{(3)} \in \mathfrak{M}$. By B-regularity, we find $M^{(1)}, M^{(2)}$ such that (29) and (30) are satisfied. If $f \in \mathcal{B}^{(\mathfrak{M})}((-1,1))$, then $f \in \mathcal{B}_{B_{0}}^{M^{(1)}}((-1,1))$ for any $B_{0}>0$. Again (i) yields the desired holomorphic approximation (since $c_{2}=C B_{0} B_{1}$ ).

The second implication in (33) follows from (ii), since [moderate growth] of $\mathfrak{M}$ yields weight sequences $N^{(i)}$ fulfilling the assumptions of (ii).

\section{Proofs}

We are now ready to prove the main results. We begin with a technical lemma in which we extract and slightly modify the essential arguments of [30, Sect. 4]. Its general formulation allows us to readily complete the pending proofs.

\subsection{A Technical Lemma}

Lemma 6.1 Let $j$ be a positive integer. Let $M^{(i)}, 1 \leq i \leq\left\lceil\log _{2}(j(j+1))\right\rceil+7=: k$, be weight sequences satisfying $\left(m_{\ell}^{(i)}\right)^{1 / \ell} \rightarrow \infty$ and

$$
\begin{array}{r}
\exists B \geq 1 \forall t>0: \bar{\Gamma}_{m^{(2)}}(B t) \leq \Gamma_{m^{(1)}}(t), \\
m g\left(M^{(i)}, M^{(i+1)}\right)<\infty, \quad \text { for } 2 \leq i \leq k-1 .
\end{array}
$$


If $f:[-1,1] \rightarrow \mathbb{C}$ is such that $f^{j}, f^{j+1} \in \mathcal{B}^{\left[M^{(1)}\right]}((-1,1))$, then $f \in$ $\mathcal{E}^{\left[M^{(k)}\right]}((-1,1))$.

Proof Set $g:=f^{j}$ and $h:=f^{j+1}$.

Let us begin with the Roumieu case. By Theorem 5.3(i), there exist families of holomorphic functions $\left(g_{\varepsilon}\right),\left(h_{\varepsilon}\right)$ approximating $g, h$, respectively. More precisely, there exist positive constants $K, c_{1}, c_{2}$ and functions $g_{\varepsilon}, h_{\varepsilon} \in \mathcal{H}\left(\Omega_{\varepsilon}\right) \cap C^{0}\left(\bar{\Omega}_{\varepsilon}\right)$ such that, for all small $\varepsilon>0$,

$$
\begin{aligned}
& \max \left\{\left\|g_{\varepsilon}\right\| \Omega_{\varepsilon},\left\|h_{\varepsilon}\right\|_{\Omega_{\varepsilon}}\right\} \leq K, \\
& \max \left\{\left\|g-g_{\varepsilon}\right\|_{[-1,1]},\left\|h-h_{\varepsilon}\right\|_{[-1,1]}\right\} \leq c_{1} h_{m^{(3)}}\left(c_{2} \varepsilon\right) .
\end{aligned}
$$

Then $g_{\varepsilon}^{j+1}-h_{\varepsilon}^{j} \in \mathcal{H}\left(\Omega_{\varepsilon}\right) \cap C^{0}\left(\bar{\Omega}_{\varepsilon}\right)$ satisfies

$$
\begin{aligned}
\left|g_{\varepsilon}^{j+1}-h_{\varepsilon}^{j}\right| & \leq\left|g_{\varepsilon}^{j+1}-f^{j(j+1)}\right|+\left|f^{j(j+1)}-h_{\varepsilon}^{j}\right| \\
& \leq(j+1) \max \left\{\left|g_{\varepsilon}\right|,|g|\right\}^{j}\left|g_{\varepsilon}-g\right|+j \max \left\{\left|h_{\varepsilon}\right|,|h|\right\}^{j-1}\left|h_{\varepsilon}-h\right| \\
& \leq c_{3} h_{m^{(3)}}\left(c_{2} \varepsilon\right), \quad \text { on }[-1,1] .
\end{aligned}
$$

Thus Lemma 5.2 implies

$$
\left\|h_{\varepsilon}^{j}-g_{\varepsilon}^{j+1}\right\|_{\Omega_{\varepsilon / 2}} \leq c_{4} h_{m^{(4)}}\left(\text { Cec }_{2} \varepsilon\right)=: \delta_{\varepsilon},
$$

where $C$ is chosen such that $C \geq \operatorname{mg}\left(M^{(i)}, M^{(i+1)}\right.$ ) for all $2 \leq i \leq k-1$. (Here and below all constants $c_{i}$ are independent of $\varepsilon$.)

Consider the continuous function

$$
u_{\varepsilon}:=\varphi_{\varepsilon} \frac{\bar{g}_{\varepsilon} h_{\varepsilon}}{\max \left\{\left|g_{\varepsilon}\right|, r_{\varepsilon}\right\}^{2}}, \quad \text { with } r_{\varepsilon}:=\delta_{\varepsilon}^{\frac{1}{j+1}}
$$

where $\varphi_{\varepsilon}$ is a smooth function compactly supported in $\Omega_{\varepsilon}$ and 1 on $\Omega_{\varepsilon / 2}$. It coincides with $h_{\varepsilon} / g_{\varepsilon}$ in $\Omega_{\varepsilon / 2} \cap\left\{\left|g_{\varepsilon}\right|>r_{\varepsilon}\right\}$, but is not holomorphic everywhere near $[-1,1]$. By taking $\varepsilon>0$ sufficiently small, we may assume that $\delta_{\varepsilon} \leq r_{\varepsilon} \leq 1$.

Lemmas 4.2.1 to 4.2.4 in [30] (which apply without change to our situation) lead to a holomorphic approximation $\left(f_{\varepsilon}\right)$ of $f$ by solving a suitable $\bar{\partial}$-problem. Indeed, they show (using (36), (37), (38) and $h_{m^{(3)}}(t) \leq h_{m^{(4)}}(e C t / 2)$, by (26) since $\left.h_{m^{(4)}} \leq 1\right)$ that

$$
\begin{aligned}
\left\|u_{\varepsilon}\right\|_{\Omega_{\varepsilon / 2}} & \leq(2 K)^{1 / j}, \\
\left\|f-u_{\varepsilon}\right\|_{[-1,1]} & \leq c_{5} r_{\varepsilon}^{1 / j},
\end{aligned}
$$

and that the bounded continuous function

$$
v_{\varepsilon}(z):=\frac{1}{2 \pi i} \int_{\Omega_{\varepsilon / 2}} \frac{\bar{\partial} u_{\varepsilon}(\zeta)}{\zeta-z} d \zeta \wedge d \bar{\zeta},
$$


which satisfies $\bar{\partial} v_{\varepsilon}=\bar{\partial} u_{\varepsilon} \mathbf{1}_{\Omega_{\varepsilon / 2}}$ in the distributional sense in $\mathbb{C}$, fulfills

$$
\left\|v_{\varepsilon}\right\|_{\Omega_{\varepsilon / 2}} \leq c_{6} \delta_{\varepsilon}^{1 / s}
$$

where $s$ is any real number with $s>j(j+1)$ (with $c_{6}$ depending on $s$ ).

Then $f_{\varepsilon}:=u_{2 \varepsilon}-v_{2 \varepsilon}$ is holomorphic in $\Omega_{\varepsilon}$ and continuous on $\mathbb{C}$. By (39) and (41), $\left\|f_{\varepsilon}\right\|_{\Omega_{\varepsilon}}$ is uniformly bounded for all small $\varepsilon$, and by (40) and (41),

$$
\left\|f-f_{\varepsilon}\right\|_{[-1,1]} \leq c_{7} \delta_{2 \varepsilon}^{1 / s} .
$$

Put $s:=2^{k-6}=: 2^{\ell}$. A repeated application of (26) gives

$$
h_{m^{(4)}}(t)^{1 / s} \leq h_{m^{(k-2)}}\left((C e)^{\ell} t\right), \quad t>0
$$

Thus, for all small $\varepsilon$,

$$
\begin{aligned}
\left\|f-f_{\varepsilon}\right\|_{[-1,1]} \leq c_{7} \delta_{2 \varepsilon}^{1 / s} & =c_{7}\left(c_{4} h_{m^{(4)}}\left(2 e C c_{2} \varepsilon\right)\right)^{1 / s} \\
& \leq c_{7} c_{4}^{1 / s} h_{m^{(k-2)}}\left(2 c_{2}(e C)^{\ell+1} \varepsilon\right) .
\end{aligned}
$$

So Theorem 5.3(ii) implies that $f \in \mathcal{E}^{\left\{M^{(k)}\right\}}((-1,1))$. This ends the proof in the Roumieu case.

For the Beurling we observe that, by assumption, we find for any (small) $c_{2}>0$ approximating sequences $\left(g_{\varepsilon}\right),\left(h_{\varepsilon}\right)$ such that (36) and (37) are satisfied. Then follow the above proof until the end and notice that thus also in the final approximation (42) the constant $2 c_{2}(e C)^{\ell+1}$ gets arbitrarily small as $c_{2}$ gets small. Again an application of Theorem 5.3 completes the proof.

\subsection{Proof of Theorem $4.2-{ }_{1} \mathcal{E}^{[\mathfrak{M}]}$}

We may assume that there is a positive integer $j$ such that $g=f^{j}, h=f^{j+1}$ are elements of the ring ${ }_{1} \mathcal{E}^{[\mathfrak{M}]}$. By composing with suitable linear reparameterizations, we may further assume that they are represented by elements of $\mathcal{B}^{[\mathfrak{M}]}((-1,1))$ which we denote by the same symbols.

In the Roumieu case, there exists $M^{(1)} \in \mathfrak{M}$ such that $g, h$ are contained in $\mathcal{B}^{\left\{M^{(1)}\right\}}((-1,1))$ (by the linear order of $\left.\mathfrak{M}\right)$. By R-regularity and R-moderate growth of $\mathfrak{M}$, we find sequences $M^{(i)} \in \mathfrak{M}$ satisfying the assumptions of Lemma 6.1 which implies that $f \in \mathcal{E}^{\left\{M^{(k)}\right\}}((-1,1))$.

In the Beurling case, we fix an arbitrary $M \in \mathfrak{M}$ and we show that $f \in$ $\mathcal{E}^{(M)}((-1,1))$. By B-regularity and B-moderate growth of $\mathfrak{M}$, we now get sequences $M^{(i)} \in \mathfrak{M}$ as required in Lemma 6.1, where $M^{(k)}=M$. By assumption, $g, h$ are elements of $\mathcal{B}^{\left(M^{(1)}\right)}((-1,1))$. Thus Lemma 6.1 gives $f \in \mathcal{E}^{(M)}((-1,1))$. 


\subsection{Proof of Theorem $3.1-{ }_{1} \mathcal{E}^{[\omega]}$}

This is an immediate corollary of Theorem 4.2 and the discussion in Sect. 4.5.

\subsection{Proof of Theorem 3.2-Non-quasianalytic ${ }_{d} \mathcal{E}^{[\omega]}$}

We reduce the multidimensional result to the one-dimensional one.

In the Roumieu case ${ }_{d} \mathcal{E}^{\{\omega\}}$, Theorem 3.2 is a simple corollary of Theorem 4.3; the weight matrix $\mathfrak{S}$ from Sect. 4.5 clearly satisfies (23) (since $\omega$ is non-quasianalytic).

The Beurling case ${ }_{d} \mathcal{E}^{(\omega)}$ can be reduced to the Roumieu case by means of the following lemma (which is an adaptation of [21, Lemma 13]).

Lemma 6.2 Let $\omega$ be a non-quasianalytic concave weight function. Suppose that $f$ : $[0, \infty) \rightarrow[0, \infty)$ is any function satisfying $\omega(t)=o(f(t))$ as $t \rightarrow \infty$. Then there exists a non-quasianalytic concave weight function $\tilde{\omega}$ satisfying $\omega(t)=o(\tilde{\omega}(t))$ and $\tilde{\omega}(t)=o(f(t))$ as $t \rightarrow \infty$.

Proof It suffices to extract some constructions from the proof of [21, Lemma 13] (to which we refer for details). We may assume that $\omega$ is of class $C^{1}$. The condition $\omega(t)=o(t)$ as $t \rightarrow \infty$ implies that $\omega^{\prime}(t) \searrow 0$ as $t \rightarrow \infty$.

Note that $\log (t)=o(\omega(t))$ and $\omega(t)=o(f(t))$ imply $f(t) \rightarrow \infty$ as $t \rightarrow \infty$. We define inductively three sequences $\left(x_{n}\right),\left(y_{n}\right)$, and $\left(z_{n}\right)$ with $x_{1}=y_{1}=z_{1}=0$, $x_{2}>0$, and the following properties:

$$
\begin{aligned}
& \int_{x_{n}}^{\infty} \frac{\omega(t)}{1+t^{2}} d t \leq \frac{1}{n^{3}}, \\
& x_{n}>2 y_{n-1}+n, \\
& f(t) \geq n^{2} \omega(t), \quad \text { for all } t \geq x_{n}, \\
& \omega\left(x_{n}\right) \geq 2^{n-i} \omega\left(z_{i}\right), \quad 1 \leq i \leq n-1, \\
& \omega^{\prime}\left(y_{n}\right)=\frac{n-1}{n} \omega^{\prime}\left(x_{n}\right), \\
& \omega\left(z_{n}\right)=n \omega\left(y_{n}\right)-(n-1)\left(\omega\left(x_{n}\right)+\left(y_{n}-x_{n}\right) \omega^{\prime}\left(x_{n}\right)\right) .
\end{aligned}
$$

Concavity of $\omega$ guarantees well-definedness of these conditions. Then

$$
\tilde{\omega}(t):= \begin{cases}(n-1)\left(\omega\left(x_{n}\right)+\left(t-x_{n}\right) \omega^{\prime}\left(x_{n}\right)\right)-\sum_{i=1}^{n-2} \omega\left(z_{i+1}\right) & \text { if } x_{n} \leq t<y_{n}, \\ n \omega(t)-\sum_{i=1}^{n-1} \omega\left(z_{i+1}\right) & \text { if } y_{n} \leq t<x_{n+1}\end{cases}
$$

defines a non-quasianalytic concave weight function of class $C^{1}$ satisfying

$$
(n-2) \omega(t) \leq \tilde{\omega}(t) \leq n \omega(t), \quad \text { if } t \in\left[x_{n}, x_{n+1}\right) \text { and } n \geq 2 .
$$

(Non-quasianalyticity follows from (43) and the second inequality in (49); cf. [21, Remark 14].) Together with (45) this implies that $\omega(t)=o(\tilde{\omega}(t))$ and $\tilde{\omega}(t)=o(f(t))$ as $t \rightarrow \infty$. 
Suppose that $g=f^{j}, h=f^{j+1}$ are representatives (of the corresponding germs) belonging to $\mathcal{B}^{(\omega)}(U)$ on some relatively compact 0 -neighborhood $U$ in $\mathbb{R}^{d}$ and consider the sequence $L_{k}$ defined in (14). Then for each integer $j \geq 1$ there exists $C_{j}>1$ such that

$$
L_{k} \leq C_{j} \exp \left(j \varphi_{\omega}^{*}(k / j)\right), \quad \text { for all } k \in \mathbb{N}
$$

Defining the function $\ell:[0, \infty) \rightarrow \mathbb{R}$ by

$$
\ell(t):=\log \max \left\{L_{k}, 1\right\}, \quad \text { for } k \leq t<k+1,
$$

and performing the subsequent steps in [21, Sect. 5], we find that $\ell \leq \varphi_{\tilde{\omega}}^{*}+$ const, where $\tilde{\omega}$ is the weight function provided by Lemma 6.2. This means that $g, h$ belong to $\mathcal{B}^{\{\tilde{\omega}\}}(U)$. Invoking Theorem 3.2 in the Roumieu case shows that $f \in{ }_{d} \mathcal{E}^{\{\tilde{\omega}\}}$. Since $\omega(t)=o(\tilde{\omega}(t))$ as $t \rightarrow \infty$ we may conclude that $f \in{ }_{d} \mathcal{E}^{(\omega)}$.

\subsection{Proof of Theorem 2.7-Quasianalytic ${ }_{d} \mathcal{E}^{\{M\}}$}

The following lemma is a variant of [16, Theorem 1.6(3)].

Lemma 6.3 Let $M$ be a quasianalytic intersectable weight sequence. Then:

(i) $n_{k}^{1 / k} \rightarrow \infty$ for all $N \in \mathcal{L}(M)$.

(ii) If $M$ has moderate growth, then for every $N \in \mathcal{L}(M)$ there exists $N^{\prime} \in \mathcal{L}(M)$ such that $m g\left(N^{\prime}, N\right)<\infty$.

Proof (i) is obvious, since $m_{k}^{1 / k} \rightarrow \infty$ (cf. Sect. 2.8).

(ii) If $M$ has moderate growth, then so has $m$. Set $C:=\operatorname{mg}(m, m)<\infty$. For $N \in \mathcal{L}(M)$ we define $N^{\prime}$ by $n_{k}^{\prime}:=C^{k} \min _{0 \leq j \leq k} n_{j} n_{k-j}$ or equivalently

$$
n_{2 j}^{\prime}:=C^{2 j} \underline{v}_{1}^{2} \underline{v}_{2}^{2} \underline{v}_{3}^{2} \cdots \underline{v}_{j}^{2}, \quad n_{2 j+1}^{\prime}:=C^{2 j+1} \underline{v}_{1}^{2} \underline{v}_{2}^{2} \underline{v}_{3}^{2} \cdots \underline{v}_{j}^{2} \underline{v}_{j+1},
$$

where $\underline{v}_{k}:=n_{k} / n_{k-1}$. Then clearly $\operatorname{mg}\left(N^{\prime}, N\right)<\infty$. Since $\underline{v}_{k}$ is increasing, so is $\underline{v}_{k}^{\prime}:=n_{k}^{\prime} / n_{k-1}^{\prime}$, thus $n^{\prime}$ is log-convex. Moreover,

$$
n_{2 j}^{\prime}=C^{2 j} n_{j}^{2} \geq \frac{m_{2 j}}{m_{j}^{2}} n_{j}^{2} \geq m_{2 j}
$$

and analogously $n_{2 j+1}^{\prime}=C^{2 j+1} n_{j} n_{j+1} \geq m_{2 j+1}$, so that $N^{\prime} \geq M$. It remains to check that $N^{\prime}$ is non-quasianalytic. Since $N^{\prime}$ is log-convex, the sequence $\left(N_{k}^{\prime}\right)^{1 / k}$ is increasing and so it suffices to show that $\sum_{j}\left(N_{2 j}^{\prime}\right)^{-1 /(2 j)}<\infty$. This is clear, since

$$
\left(N_{2 j}^{\prime}\right)^{1 /(2 j)}=\left((2 j) ! C^{2 j} n_{j}^{2}\right)^{1 /(2 j)} \geq \frac{2 C}{e} j n_{j}^{1 / j} \geq \frac{2 C}{e} N_{j}^{1 / j}
$$

and $N$ is non-quasianalytic. 
Let $M$ be a quasianalytic intersectable weight sequence of moderate growth. Suppose that $g=f^{j}, h=f^{j+1}$ are elements of ${ }_{d} \mathcal{E}^{\{M\}}$. Since $M$ is intersectable, it suffices to show that $f \in{ }_{d} \mathcal{E}^{\{N\}}$ for every $N \in \mathcal{L}(M)$. Fix such $N$. By Lemma 6.3, there exist $N^{(1)}, \ldots, N^{(k)} \in \mathcal{L}(M)$ with $N^{(k)}=N$ such that the requirements of Lemma 6.1 are satisfied. (Note that $\mathcal{L}(M)$ is not a weight matrix in the sense of Sect. 4.1, because it is not totally ordered.)

Let $U$ be an open 0-neighborhood in $\mathbb{R}^{d}$ on which we have $g, h \in \mathcal{E}^{\{M\}}(U)$ for representatives which are denoted by the same symbols. Take any curve $c \in \mathcal{E}^{\left\{N^{(1)}\right\}}(\mathbb{R}, U)$ with compact support. Then, by composition closedness of $\mathcal{E}^{\left\{N^{(1)}\right\}}$ as $n^{(1)}$ is log-convex, we have $g \circ c, h \circ c \in \mathcal{E}^{\left\{N^{(1)}\right\}}(\mathbb{R})$. After a linear change of variables, we may assume that $g \circ c, h \circ c \in \mathcal{B}^{\left\{N^{(1)}\right\}}((-1,1))$. Thus Lemma 6.1 yields that $f \circ c \in \mathcal{E}^{\{N\}}((-1,1))$. This implies that $f \in \mathcal{E}^{\{N\}}(U)$, by [16, Theorem 2.7].

Acknowledgements We wish to thank Prof. José Bonet Solves for drawing our attention to [30].

Funding Open access funding provided by University of Vienna.

Open Access This article is licensed under a Creative Commons Attribution 4.0 International License, which permits use, sharing, adaptation, distribution and reproduction in any medium or format, as long as you give appropriate credit to the original author(s) and the source, provide a link to the Creative Commons licence, and indicate if changes were made. The images or other third party material in this article are included in the article's Creative Commons licence, unless indicated otherwise in a credit line to the material. If material is not included in the article's Creative Commons licence and your intended use is not permitted by statutory regulation or exceeds the permitted use, you will need to obtain permission directly from the copyright holder. To view a copy of this licence, visit http://creativecommons.org/licenses/by/4.0/.

\section{References}

1. Amemiya, I., Masuda, K.: On Joris' theorem on differentiability of functions. Kodai Math. J. 12(1), 92-97 (1989)

2. Boman, J.: On the intersection of classes of infinitely differentiable functions, Ark. Mat. 5 (1963/1965), 301-309

3. Boman, J.: Differentiability of a function and of its compositions with functions of one variable. Math. Scand. 20, 249-268 (1967)

4. Bonet, J., Meise, R., Melikhov, S.N.: A comparison of two different ways to define classes of ultradifferentiable functions. Bull. Belg. Math. Soc. Simon Stevin 14, 424-444 (2007)

5. Braun, R.W., Meise, R., Taylor, B.A.: Ultradifferentiable functions and Fourier analysis. Results Math. 17(3-4), 206-237 (1990)

6. Chaumat, J., Chollet, A.-M.: Surjectivité de l'application restriction à un compact dans des classes de fonctions ultradifférentiables. Math. Ann. 298(1), 7-40 (1994)

7. Duncan, J., Krantz, S.G., Parks, H.R.: Nonlinear conditions for differentiability of functions. J. Anal. Math. 45, 46-68 (1985)

8. Dyn'kin, E.M.: Pseudoanalytic extension of smooth functions. The uniform scale. Transl. Ser. 2, Am. Math. Soc. 115 (1980), 33-58 (English)

9. Fürdös, S., Nenning, D.N., Rainer, A., Schindl, G.: Almost analytic extensions of ultradifferentiable functions. J. Math. Anal. Appl. 481(1) (2020). https://doi.org/10.1016/j.jmaa.2019.123451

10. Jaffe, E.Y.: Pathological phenomena in Denjoy-Carleman classes. Can. J. Math. 68(1), 88-108 (2016)

11. Joris, H.: Une $\mathcal{C}^{\infty}$-application non-immersive qui possède la propriété universelle des immersions. Arch. Math. (Basel) 39(3), 269-277 (1982)

12. Joris, H., Preissmann, E.: Pseudo-immersions. Ann. Inst. Fourier (Grenoble) 37(2), 195-221 (1987)

13. Joris, H., Preissmann, E.: Quotients of smooth functions. Kodai Math. J. 13(2), 241-264 (1990) 
14. Komatsu, H.: An analogue of the Cauchy-Kowalevsky theorem for ultradifferentiable functions and a division theorem for ultradistributions as its dual. J. Fac. Sci. Univ. Tokyo Sect. IA Math. 26(2), 239-254 (1979)

15. Kriegl, A., Michor, P.W., Rainer, A.: The convenient setting for non-quasianalytic Denjoy-Carleman differentiable mappings. J. Funct. Anal. 256, 3510-3544 (2009)

16. Kriegl, A., Michor, P.W., Rainer, A.: The convenient setting for quasianalytic Denjoy-Carleman differentiable mappings. J. Funct. Anal. 261, 1799-1834 (2011)

17. Mandelbrojt, S.: Séries adhérentes, régularisation des suites, applications. Gauthier-Villars, Paris (1952)

18. Matsumoto, W.: Characterization of the separativity of ultradifferentiable classes. J. Math. Kyoto Univ. 24(4), 667-678 (1984)

19. Rainer, A.: Arc-smooth functions on closed sets. Compos. Math. 155, 645-680 (2019)

20. Rainer, A.: Quasianalytic ultradifferentiability cannot be tested in lower dimensions. Bull. Belg. Math. Soc. Simon Stevin 26, 505-517 (2019)

21. Rainer, A.: On the extension of Whitney ultrajets of Beurling type. Results Math. 76, 36 (2021). https:// doi.org/10.1007/s00025-021-01347-z

22. Rainer, A., Schindl, G.: Composition in ultradifferentiable classes. Stud. Math. 224(2), 97-131 (2014)

23. Rainer, A., Schindl, G.: Equivalence of stability properties for ultradifferentiable function classes. Rev. R. Acad. Cienc. Exactas Fis. Nat. Ser. A Math. RACSAM. 110(1), 17-32 (2016)

24. Rainer, A., Schindl, G.: On the extension of Whitney ultrajets. Stud. Math. 245(3), 255-287 (2019)

25. Rainer, A., Schindl, G.: On the extension of Whitney ultrajets II. Stud. Math. 250(3), 283-295 (2020)

26. Rudin, W.: Division in algebras of infinitely differentiable functions. J. Math. Mech. 11, 797-809 (1962)

27. Schindl, G.: Exponential laws for classes of Denjoy-Carleman differentiable mappings, Ph.D. thesis, Universität Wien, 2014, http://othes.univie.ac.at/32755/1/2014-01-26_0304518.pdf

28. Schmets, J., Valdivia, M.: On certain extension theorems in the mixed Borel setting, J. Math. Anal. Appl. 297(2), 384-403 (2003). Special issue dedicated to John Horváth

29. Thilliez, V.: Smooth solutions of quasianalytic or ultraholomorphic equations. Monatsh. Math. 160(4), $443-453$ (2010)

30. Thilliez, V.: Functions with ultradifferentiable powers. Results Math. 75(3), 79 (2020)

Publisher's Note Springer Nature remains neutral with regard to jurisdictional claims in published maps and institutional affiliations. 